\title{
DE QUELQUES TEMPORALITÉS DE LA RÉCEPTION TÉLÉVISUELLE'
}

\author{
Gisèle Bertrand ${ }^{2}$, Gérard Derèze ${ }^{3}$ et Pierre-Alain Mercier ${ }^{4}$
}

La question que nous souhaitons aborder dans cet article peut être ainsi schématisée: comment s'articulent, se combinent, s'échangent, se mélangent, se contaminent, se co-construisent les temporalités télévisuelles, essentiellement dans leurs dimensions réceptives, et celles de la vie quotidienne.

Nombreuses sont les problématiques que traverse cette question: quant à la logique du récit, quant aux modalités de structuration et de représentation du temps, de son écoulement, de sa scansion, de sa mémoire; quant à son utilisation (entre autres dans les approches de type "budget-temps"); quant à la fictionnalisation du réel et vice versa; quant à la distinction qu'il faut désormais opérer entre "temps vrai" (selon l'expression de Virilio) ou "temps vécu" et "temps réel" (celui du média).

Nous allons, pour notre part, tenter ici d'envisager cette question selon deux modalités distinctes et pourtant reliées. Avant d'explorer

1 Le présent article reprend, pour partie, des éléments présentés dans un rapport INACNRS (Contrat de définition $n^{\circ} 956297$, Paris, novembre 1994).

2 Directeur d'études à l'INA (Institut National de l'Audiovisuel), Paris.

3 Professeur adjoint au Département de communication de l'Université catholique de Louvain.

4 Sociologue à l'Iris -TS, Cnrs, Université de Paris IX-Dauphine. 
dans la deuxième partie de ce texte ("Temporalités conçues") ce que proposent quelques approches théoriques développées dans la perspective de la prise en compte de la dimension réceptive par les pôles de production, il nous semble intéressant de fournir quelques éléments sur les diverses façons dont les téléspectateurs eux-mêmes posent le problème de la relation entre temps (en général, mais aussi, en particulier -le temps de la vie domestique-) et télévision. Pour ce faire, nous repartirons, dans la première partie de cet article ("Temporalités vécues"), des résultats de deux recherches empiriques, menées l'une en France et l'autre en Belgique.

L'objectif que nous poursuivons, dans les pages qui suivent, n'est en aucun cas de fournir au lecteur un cadre englobant (totalisant ou "méta") ayant l'ambition de prendre en compte la multiplicité des points de vue et des angles d'approche. Nous proposons plutôt, au travers de deux plongées empiriques et d'une mise en perspective compréhensive de certaines propositions théoriques, de dégager quelques axes -non nécessairement homogènes- d'interprétation possible et d'apporter quelques éléments de cadrage permettant de mettre en œuvre un processus de confrontation dynamique et, pour ainsi dire, herméneutique. Le lecteur aura à établir des relations, à opérer des distinctions, à croiser -à la lumière de ses propres expériences ou travaux-les pistes et les interprétations.

\section{Temporalités vécues}

Souhaitant donner une place importante et initiale à quelques éléments réflexifs et interprétatifs implantés et construits dans le mouvement de démarches empiriques, une double opportunité s'est offerte à nous. En premier lieu, nous tenterons de réexploiter un terrain constitué autour d'un questionnement plus large ${ }^{1}$ portant sur la façon dont la technicisation de l'environnement quotidien est concrètement vécue par des ménages, en sachant que dans le processus de

1 Il s'agit d'une recherche comparative menée depuis 1992 par J. Coutras, J.L. Lacascade, P.-A. Mercier et R. Tourreau, en coopération avec S. Meyer et E. Schulze (Technische Universität de Berlin), ayant pour objet de montrer et d'expliquer la diversité, la spécificité et l'évolution de l'articulation entre équipement technique des foyers et cultures familiales. Dans ce but, l'équipe française a réalisé, en 1993 et 1994, 40 entretiens en profondeur auprès de ménages parisiens appartenant aux "nouvelles couches moyennes". 
technicisation, l'acquisition de la télévision puis le développement de la consommation des programmes tiennent une place non négligeable. Ensuite, dans un second temps, la recherche sur laquelle nous allons nous baser s'est effectuée en Belgique francophone et s'est particulièrement attachée à l'étude des objets médiatiques (journaux, radio, télévision -et leurs extensions-) utilisés, par des personnes de plus de 55 ans professionnellement inactives, dans le cadre de leur unité de résidence, cet espace où "tout porte témoignage"1.

\subsection{Des différents termes de la réception}

Malgré les limites imposées par l'exercice même de relecture ${ }^{2}$, les données du terrain français apportent des informations intéressantes sur la façon dont des téléspectateurs ordinaires conçoivent et perçoivent le problème que nous posons. La pertinence de ce travail apparaît d'ailleurs très globalement lorsque nous constatons que, dans la grande majorité des cas, la question de la télévision est d'abord -et, quelquefois, uniquement- abordée en termes de temps plutôt que de programmes: qu'il s'agisse de pratiques (quand regarde-t-on la télévision, quand l'a-t-on acquise?), de jugements -le temps (excessif) qu'elle prend- ou de fonctions (comme celle, attribuée au magnétoscope, de "mise en conserve" du temps).

1 F. ZONABEND, "La parenté: origines et méthodes de la recherche; usages sociaux de la parenté", Ethnologies en miroir (Essais réunis par I. CHIVA, U. JEGGLE), Paris, Ed. MSH, 1987, p. 106.

2 Le choix de réinterroger (voire de détourner) un terrain qui n'a pas été conçu pour le présent projet, mais qui apporte des informations sur les rapports entre vie quotidienne et télévision, induit un intérêt et des limites spécifiques. L'intérêt réside essentiellement dans le fait même que le problème qui nous occupe -celui du mode d'articulation entre les deux ordres de temporalité-était tout à fait périphérique à la problématique constitutive du terrain. De ce fait, la façon qu'ont eue les interviewés de poser et d'exprimer la question de l'articulation entre les deux ordres de temporalité a été d'autant plus spontanée qu'elle n'a pas été "sollicitée" par le questionnement. En revanche, la dominante d'une thématique en termes de technicisation et d'équipement tend à donner une importance très grande à certaines dimensions accessoires de notre point de vue, ainsi le caractère "d'objet technique" de la télévision ne nous intéresse-t-il que secondairement. Par ailleurs l'intérêt porté aux problèmes d'équipement conduit parfois les interviewés à privilégier l'objet télévision par rapport aux programmes ainsi que le long ou, du moins, le moyen terme (dans lesquels s'inscrivent les logiques d'équipement, de renouvellement, bref de "consommation" de biens) par rapport au court terme, à la quotidienneté, a priori plus pertinents en termes de "consommation" de programme. 
Nous présentons les éléments issus de cette relecture selon un principe simple, opératoire -donc, de ce fait même, contestable-: en fonction de la structuration des différentes strates de temporalité entre long, moyen et court termes (ce qui pose le problème de la définition nécessairement arbitraire des frontières entre ces strates), croisée par la dimension individuelle, interne au foyer, ou sociale des représentations de ces strates. Cette présentation est d'autant plus schématique que les problèmes d'articulation entre les temporalités risquent fort de se situer aux marges et aux croisements des différentes dimensions déclinées.

\section{a. Le long terme individuel}

L'acquisition de la télévision, son renouvellement, ou bien encore l'acquisition d'un magnétoscope apparaissent comme des repères temporels dans le déroulement de la vie du téléspectateur et de sa famille. En tant que telle, la télévision -comme objet, mais aussi, quelquefois, comme programmes- est donc souvent intimement liée à l'histoire de la famille.

Ce lien peut être précisément datable: l'arrivée de la télévision dans la vie familiale est référée à un événement particulier et important de cette dernière. L'objet "téléviseur" a pu être un cadeau célébrant cet événement: cadeau (ou acquisition à l'occasion) de mariage, cadeau de Noël, voire d'anniversaire. Plus fréquemment, l'entrée dans le temps de la télévision (par l'acquisition d'un premier poste) coïncide avec une naissance d'enfant qui se traduit par un changement sensible dans le mode de vie du ménage, qui se "recentre" en quelque sorte autour de l'enfant. Un exemple montre de façon particulièrement imagée ce lien entre un changement de situation personnelle et l'arrivée de la télévision dans l'univers domestique -celui de la coïncidence entre la grossesse se traduisant par une immobilisation de la femme et l'adoption de la télévision-: "j'avais loué une télévision pour faire passer à ma femme les après-midi où elle attendait les enfants, les jumeaux, elle était astreinte à un certain immobilisme".

Plus tard, la croissance des enfants, devenant eux aussi consommateurs, se traduira souvent par le passage au multi-équipement et/ou par l'acquisition d'un magnétoscope.

Plus largement la télévision, non seulement dans son acquisition, mais aussi et surtout dans son usage, dans la place qu'elle prend dans la vie quotidienne du ménage, dans l'empreinte qu'elle y laisse, marque les grandes périodes du cycle de vie. Il y a des périodes où 
l'on est "plus ou moins télévision". Dans plusieurs cas, est exprimée une relation quasi-mécanique entre l'usage de la télévision et le temps que l'on passe globalement chez soi, et qui varie beaucoup en fonction des différentes périodes de la vie. Ainsi la période ante-télévision, période avant l'arrivée des enfants ${ }^{1}$ ou, plus largement, avant l"'installation" véritable du ménage, est-elle, de ce fait, souvent l'objet d'une évocation nostalgique: l'âge que l'on avait lorsqu'"elle" est arrivée, l'évolution, souvent marquée par la substitution de la pratique télévisuelle à des activités qu'on a abandonnées -substitution quelquefois anticipée ("on la regardera quand on sera plus vieux peut-être")ou bien, au contraire, lassitude et progressif abandon de la pratique sur une longue période ("moi j'ai beaucoup regardé la télévision quand j'étais jeune mais aujourd'hui je suis devenu assez peu télévision").

Cette coïncidence entre les périodes "télévision" et les grands cycles de la vie individuelle et familiale est la façon la plus fréquente de conjuguer au long terme cette inscription du "temps du média" dans celui de la vie domestique. D'autres associations apparaissent sur un mode plus mineur: à travers le téléviseur lui-même, par son transport d'une adresse à une autre, ou bien par sa transmission inter-générationnelle (cadeau des enfants à leurs parents âgés ou bien, au contraire, cadeau ou héritage venant des parents), la télévision semble parfois relier symboliquement différentes époques ou différentes générations; à travers les pratiques d'enregistrement, le magnétoscope se conjugue aussi quelquefois avec cette temporalité à long terme du ménage, outil symbolique de reproductibilité par rapport à la vie qui va et qui ne va qu'une fois; enfin le vieillissement-dégradation ou simple obsolescence- du matériel renvoie indirectement à celui des membres du ménage, ainsi la déportation du vieux poste noir et blanc dans la maison de campagne (que l'on vient parfois d'acquérir ou de commencer à meubler) semble-t-elle indissociable de la révolution de toute une époque familiale.

A travers la télévision, certains événements d'actualité s'inscrivent dans l'histoire familiale: ils constituent des repères appropriables au déroulement de sa propre histoire: c'est ainsi qu'un interviewé

1 Les problèmes de garde des très jeunes enfants semblent souvent faire de ces années des années "très télévision" -pour les parents-, le temps consacré au média pouvant parfois diminuer ensuite, avec la croissance des enfants, ce qui n'implique pas nécessairement une décroissance globale de la place de la télévision dans la famille, les enfants, à leur tour, étant devenus consommateurs de programmes. 
exprime une coïncidence quasi-mnémotechnique entre son rapport à la télévision, une situation précise de son histoire personnelle et le début de la guerre au Liban. Ce croisement dans le long terme, à travers la télévision, entre la "petite" et la "grande" histoire introduit l'idée de la dimension "sociale" de ce vécu temporel de la télévision.

\section{b. Le long terme social}

Comme objet de consommation, comme objet technique et comme programmes, la télévision constitue également un repère qui permet de synchroniser le temps interne du foyer à celui d'un environnement plus ou moins large.

C'est d'abord très évidemment le cas lorsque l'acquisition a concordé avec un événement historique ou, plus précisément, un "événement historico-médiatique". Dans les années 50, on a ainsi pu s'équiper à l'occasion du couronnement de la Reine d'Angleterre; à la fin des années 60 pour voir en direct les premiers pas de l'homme sur la lune; plus récemment les Jeux Olympiques (de Séoul) semblent avoir constitué l'occasion d'acheter ou de renouveler le téléviseur, de passer à la couleur ou, plus fréquemment encore, d'acquérir un magnétoscope.

A travers les pratiques d'enregistrement, le couple télévisionmagnétoscope apparaît maintenant comme l'outil privilégié d'une recherche du temps perdu, voire une machine à remonter le temps. Le magnétoscope est parfois acquis et utilisé dans l'espoir (utopique ?) de laisser aux enfants, aux générations ultérieures, par l'enregistrement de documents sur l'histoire immédiate, un témoignage sur ce qu'était le contexte, l'époque et l'histoire qui ont baigné la relative jeunesse des futurs ancêtres. D'autres, comme ce magnétophile qui enregistre systématiquement tous les documents sur les chanteurs des années 60 pour se constituer une vidéothèque personnelle sur ce thème, se livrent ainsi à une tentative de stockage, de "capitalisation" du temps qui passe, capitalisation souvent au second degré puisque les documents enregistrés sont, pour leur grande majorité, déjà des documents d'archives.

Dans une conception plus sociologique du contexte, de l'Histoire, c'est la télévision elle-même, entendue comme ensemble meuble/technique/programme qui constitue le repère d'une époque, d'une certaine couleur du temps: "mes enfants ont vécu, certains dès leur plus jeune âge, dans le bain télévision, c'est-à-dire qu'ils voyaient 
Belphégor, ils en parlent encore maintenant, quand ils parlent de soirées de télévision où ils étaient accrochés à..."

Télévision et magnétoscope sont perçus, en fonction de leur apparence et de leur degré de sophistication, comme des repères temporels en termes de technique ou de design. Pour l'un, le passage du noir et blanc à la couleur marque plus largement une entrée dans un certain "modernisme". Tel autre conserve son poste noir et blanc parce que c'est un classique du design, exposé au Moma de New York. Le fait même de posséder un téléviseur, passant du luxe à l'équipement de base, symbolise pour certains l'évolution des mœurs et de la consommation. Et l'envahissement progressif du temps quotidien (ici, celui des autres) par la télévision paraît refléter une évolution socio-culturelle plus large, récente, et plutôt inquiétante. En ce sens les pratiques télévisuelles sont appréhendées comme un marqueur de cette évolution.

\section{c. Le court terme individuel}

Comment la temporalité de la télévision s'inscrit-elle concrètement dans la vie quotidienne des ménages ?

Appréhendées dans le court terme, les modalités d'articulation entre les deux ordres de temporalité renvoient d'abord aux différents statuts que peut avoir l'“activité": regarder la télévision au sein des autres activités -ou inactivités- qui occupent la journée. Schématiquement, les différents modes d'articulation semblent relever des figures de la concurrence, de l'alternative, de la substitution, de l'association et de l'agglomération.

La figure de la concurrence implique une tension entre une activité et l'éventualité (perçue comme inconciliable) de regarder la télévision. Elle concerne surtout les moments que la grille des programmes situe dans le "prime time". Les diverses activités par rapport auxquelles l'écoute de la télévision s'inscrit en concurrence peuvent être, par exemple, la lecture, la vaisselle ou le coucher des enfants. Cette situation de concurrence est perçue non seulement pour soimême, mais aussi, fréquemment, pour les enfants: la pratique de la télévision semble alors constituer une concurrence généralement perçue comme néfaste (et déloyale) à d'autres occupations plus valorisées, qui peuvent être: lire, écouter ou pratiquer de la musique. Par ailleurs, l'idée de concurrence paraît parfois devoir se lire davantage en termes de "présence" que d'activité: on ne peut, en quelque sorte, être à la fois présent aux autres (membres du foyer) et "présent au 
poste"("après, bon, quand il est là, je veux pas sacrifier mon mari à la télévision, donc on coupe tout, on dîne tranquillement, et puis éventuellement après on se met un bout de cassette"; "quand on est avec la petite, on éteint la télé, on discute avec elle pendant le repas, etc. et une fois qu'elle est couchée bon on rallume la télé et à ce moment-là on en profite").

La figure de l'alternative s'exprime, elle, souvent de façon projective, par un "soit, soit"; elle n'implique pas la tension inhérente à la concurrence. Quant à la substitution, elle joue majoritairement dans le sens du remplacement d'un programme par une autre activité, soit dans une logique de rupture de routine (ce qu'un soir on décide de substituer à la norme: regarder la télévision), soit, en quelque sorte, en remplacement obligé (ce qu'il faut bien envisager les soirs où "il n'y a rien à la télé").

Alors que ces figures traduisent globalement l'impossibilité de mélanger certaines activités avec l'écoute de la télévision (donc, en un sens, l'impossibilité de combiner leurs deux types spécifiques de temporalité), l'association ou ce que nous appelons l'agglomération, partent, elles, de la position inverse et aboutissent à l'apparition dans la vie quotidienne de "temps mixtes" pour la première et de "temps intégrés" pour la seconde.

L'association combine toutes les activités que l'on peut avoir, ou continuer d'avoir tout en regardant la télévision. Ces activités (dîner, repasser-le modèle de l'association parait relativement fréquent pour les activités et les programmes de la journée-, lire le journal, faire les mots croisés, broder...) sont souvent les mêmes que celles que d'autres perçoivent en concurrence de la pratique de la télévision.

L'idée d'agglomération, elle, va plus loin. Ainsi, la façon dont certains expriment leurs pratiques quotidiennes conduit à penser qu'il y a des cas où il serait aussi arbitraire de tenter de hiérarchiser les deux activités ("regarder la télévision" et l'autre activité), voire de les séparer, qu'absurde de distinguer au sein de la conduite automobile appréhendée comme une activité en soi le fait de passer une vitesse de celui de regarder la route en même temps. L'activité réelle, celle qui devrait être recensée comme telle, serait alors "faire ceci ou cela (dîner, repasser, construire des maquettes)... en regardant la télévision": il n'y a pas juxtaposition, mais véritable intégration, fusion de deux pratiques qui en constituent une nouvelle, spécifique, irréductible à ses constituants (ce qui pose précisément le problème de la nature de la temporalité propre à ces agglomérations). 
Par ailleurs télévision et magnétoscope paraissent s'inscrire dans la structuration de la vie familiale par le biais de ce qu'on pourrait appeler une fonction programmatique. A une idée de programme (liée à la "grille" de la télévision, mais aussi à la programmation du magnétoscope, voire à la consultation du "journal de programmes") semble répondre celle d'une véritable programmation de la vie familiale et, parfois plus particulièrement, des activités des enfants (la structure de cette programmation étant associée à la grille de la télévision). Au sein de cette programmation domestico-médiatique, le "JT" joue un rôle de pivot, de marqueur de la transition d'une période du programme (tant familial que télévisuel) à une autre. Et à la notion de plage (de programmes), produit de la logique de découpage du média, semble répondre une idée d'heures dédiées (à la consommation de la télévision).

Le magnétoscope joue, bien évidemment, un rôle important dans l'adéquation entre les deux types de programmation, permettant de faire coïncider quand il le faut plages et heures dédiées ("enregistrer les programmes qui sont à 10 heures et demie et puis les repasser aux heures intéressantes..."). De façon plus générale, les pratiques d'enregistrement, de report d'écoute, permettent d'introduire de la souplesse dans la programmation domestico-médiatique, et, à l'extrême, d'inscrire le temps du média dans les "créneaux" que lui consacre le temps domestique, mais la possibilité d'un total asservissement du temps média à la temporalité domestique est précisément limitée par la fonction programmatique largement allouée à l'ensemble du système télévisuel. D'autant qu'à côté de cette fonction, télévision et magnétoscope semblent quelquefois en remplir une autre au sein du foyer, une véritable fonction horlogère: par le repère que constitue le moment pivot du JT; par l'introduction dans l'espace domestique de la rigidité du temps de l'horloge qui régit la programmation du magnétoscope; symboliquement, par la petite pendule qui orne la façade du magnétoscope; par la perception même du décalage entre le type de temps du programme et celui qui organise les activités et mouvements des membres du foyer ("J'ai un mari qui n'a pas d'heures..." dit une interviewée, et le contexte de son discours nous amène à compléter: ... contrairement à la télé).

La coïncidence fréquente entre certaines situations dans le foyer et le fait de regarder la télévision conduit par ailleurs à nous interroger sur la nature du temps consacré à la télévision. En effet certains 
travaux de l'Insee sur les budgets temps des ménages ${ }^{1}$ regroupent les temps consacrés au sommeil, aux "prises alimentaires" et aux soins corporels sous le terme générique de "temps physiologiques". Or, audelà de son association traditionnelle avec le repas du soir, la pratique de la télévision entretient un rapport suffisamment fréquent avec le sommeil pour que l'on puisse poser la question de son éventuelle appartenance à ce "temps physiologique": on s'endort devant la télévision, on la regarde -au lit- quand on est fatigué. En fait le problème est peut-être de savoir, de façon moins schématique, si le temps de la télévision se confond avec le temps physiologique ou s'il est limité par lui. Ce que l'on pourrait formuler de façon volontairement ambiguë par l'idée que le temps de la télévision touche au temps physiologique: soit il en relève presque (la télévision-sommeil, machine à se bercer, à s'endormir, la télé hypnotique), soit il en est frontalier (d'un rêve à l'autre), soit il lui dispute les mêmes heures. Dans ce dernier cas, l'utilisation du magnétoscope pour reporter la consommation des programmes de la nuit dans les heures dédiées peut permettre de réimperméabiliser la frontière entre consommation des programmes et temps physiologique. Cette frontière temporelle (à la fois passage et séparation) se double souvent d'une frontière spatiale: selon les cas, le téléviseur sera banni ou accueilli dans la chambre à coucher.

Cette association entre espace et temps domestiques se lit également dans l'espace (quantité d'espace, situation) attribué dans le foyer au téléviseur. Ainsi à titre d'exemple, à la volonté de le rendre très discret, presque invisible, est nettement associée celle de ne pas lui consacrer trop de temps. Plus largement la télévision participe des différents partages internes au foyer (qu'elle contribue, dans le même mouvement, à structurer) entre les temps, les espaces et les générations: le partage journée/soir, le partage enfants/adultes, le partage entre activités, le partage des tâches dans le couple...

\section{d. Le court terme social}

La recherche dont nous réexploitons le terrain privilégiait la vie interne au foyer, ce qui explique qu'à l'échelle du court terme la relation à la télévision ne se soit pas traduite en termes de temporalité sociale (par exemple, la façon et le temps de parler des programmes à l'extérieur du foyer, avec des voisins, des collègues). On note cepen-

1 Voir, par exemple, C. RoY, "La gestion du temps des hommes et des femmes, des actifs et des inactifs", Économie et Statistique, juillet/août 1989. 
dant chez certains interviewés jeunes appartenant à des milieux relativement intellectuels, ou, du moins, valorisant les échanges et la vie culturelle, que l'appréciation du (faible) temps consacré quotidiennement à la télévision (davantage que le choix de tel ou tel programme) est utilisée comme l'indicateur d'une proximité sociale et culturelle avec ceux que l'on fréquente. A l'extrême, la non-possession d'un téléviseur joue encore un rôle de signe, voire de connivence avec un milieu qui serait par ailleurs difficile à définir en dehors de ce critère ("nos copains, ici, à la limite, ils n'ont même pas la télé...", "... c'est une question de notre choix des amis"). C'est probablement davantage en termes d'emploi du temps qu'en termes de (faible) légitimité culturelle du média que se constitue le clivage (donc en termes de modes de vie plus qu'en termes de prétention culturelle).

Notons aussi que les problèmes de temporalité vécus dans leur foyer par les téléspectateurs ordinaires se situent quelquefois à l'intérieur même de la temporalité du média ou des programmes. On est ici à la frontière entre court terme et temps réel. C'est à cette échelle que l'on manipule quelquefois le rythme des programmes (entre autres, par le zapping) et que l'on gère leur concurrence interne (y compris la concurrence publicité/programmes) en termes d'espace -ici, espace de l'écran- et de temps. Le magnétoscope joue un rôle spécifique à ce niveau, en offrant un instrument adéquat à la gestion de la concurrence des programmes (il permet d'en regarder un tout en enregistrant un autre) et de combiner à sa guise flux et stock. Par ailleurs les pratiques de report d'écoute se situent le plus souvent à l'échelle du court ou du moyen terme ("quelquefois on dit qu'on va l'enregistrer comme ça on regardera le lendemain et tout ça, mais si on regarde pas le lendemain, après on regarde plus...").

\section{e. Le moyen terme individuel}

Cette catégorie ne peut être construite que par référence aux deux autres. On n'est plus dans le quotidien, mais pas encore dans la longue durée. Dans la perception de la temporalité attachée à la pratique de la télévision, le moyen terme se situe approximativement entre quelques jours et un mois. C'est à cette échelle que paraît pertinente l'appréciation de son propre rythme de consommation de programmes (les jours ou soirs de la semaine où "on regarde la télévision"), en oubliant le plus souvent la fréquentation quotidienne du JT, routine quasi invisible parce qu'elle ne correspond pas à l'idée de "regarder un programme". C'est également sur ce terme que se mesure le rythme d'ap- 
provisionnement (par achat et surtout par location) en vidéogrammes, que se jauge l'offre (que s'évalue le "regardable": "on voyait qu'il y avait des films qui passaient à la télé.../... que le soir on était de sortie.../... on s'est dit il y a deux trois films bien qui passent dans le mois..") et que se constituent les attentes par rapport à la télévision ("les semaines où il n'y a rien à voir", "j'aime bien les films, bon il y en a quand même pas mal sur Canal + , donc avec plusieurs chaînes on arrive à avoir des films pas tous les soirs mais enfin...") et que les pratiques fluctuent significativement (les semaines où on a très peu regardé la télévision).

C'est surtout, plus encore que le court terme, l'échelle pertinente pour envisager les pratiques liées au magnétoscope. Dans une majorité de cas, le magnétoscope permet en effet un report, une (ré)inscription de la consommation des programmes dans le cadre de la semaine. C'est à cette échelle surtout que le magnétoscope permet, en quelque sorte, de décoller du temps réel de la télévision ("en fait la télé nous sert plus de moniteur pour le magnétoscope que pour regarder vraiment ce qui y passe, parce qu'en plus avec Canal + on enregistre des tas de trucs puis on se les visionne quand on veut"). Ceci peut, selon les cas, se traduire, en termes de "budget-temps", par des reports ou bien par une augmentation du temps global consacré au média.

Par ailleurs, l'idée de programmation intégrée (c'est-à-dire à la fois de l'écoute du média et de la gestion des activités domestiques) trouve elle aussi une pertinence spécifique dans le cadre de la semaine, et ce d'autant plus lorsqu'il existe des pratiques plus ou moins systématiques et répétitives durant le week-end. Dans ce cas, en termes de programmation, le partage semaine/week-end conduit à inscrire la pratique de la télévision le week-end dans un autre type de temporalité et par rapport à un autre type de concurrence.

Enfin c'est principalement à l'échelle du moyen terme que sont perçues les limites (s'exprimant tantôt davantage en temps global et en moments -heures dédiées certains jours de la semaine- tantôt en termes de contenu des programmes) à la consommation des programmes et qu'émerge une conscience du temps passé devant l'écran, parfois à travers la connaissance de données statistiques, mais le plus souvent pour soi-même, par une sensation a posteriori de temps perdu ("je suis assez déçue des heures passées devant la télé dont j'estime qu'elles sont souvent perdues pour moi"). 


\section{f. Le moyen terme social}

Au niveau des relations avec l'environnement social, l'échelle du moyen terme apparaît peu dans les discours des téléspectateurs, si ce n'est, peut-être, dans les pratiques d'enregistrement et d'échanges de programmes enregistrés. Cet échange de programmes au sein d'un groupe (familial, vicinal, professionnel ou amical) pourrait à la limite être appréhendé comme un "échange de temps" d'un foyer à un autre.

D'après cette relecture du terrain, il apparaît que la télévision interfère avec les temps domestiques à trois niveaux qui peuvent se retrouver mêlés : le court terme, temps de la consommation du programme, le moyen terme, celui de sa gestion, le long terme, temps des repères familiaux, sociaux et historiques.

\subsection{De l'appréhension ordinaire du temps domestico- médiatique}

La deuxième opportunité de réexploitation d'une recherche empirique porte principalement sur certaines modalités par lesquelles des personnes de plus de 55 ans professionnellement inactives ${ }^{1}$ établissent leurs rapports au temps dans leurs pratiques reliées aux médias. Nous nous intéressons exclusivement ici au temps domesticomédiatique et n'entrons donc pas dans les manières globales et différentielles de penser (et de construire) le temps. Nous nous bornons à envisager comment les rapports des informateurs ${ }^{2}$ à la télévision construisent, dans un processus dialectique, une appréhension du temps domestique. Nous sommes conscient du caractère partiel et subjectif de cette perspective qui isole le champ domestico-média-

1 Pour plus de précisions, voir G. DERÈze, Une ethnosociologie des objets domestico-médiatiques. Médias, quotidien et troisième âge, Louvain-la-Neuve, CIACO, 1994. La problématique centrale de cette recherche peut se résumer comme suit: "Quels sont les rôles et les fonctions que les personnes du troisième âge (ou autrement dit les personnes de plus de 55 ans, professionnellement inactives) attribuent et font jouer aux objets domestico-médiatiques (journaux et revues, radio, télévision) dans leur vie quotidienne?" La recherche ne s'est pas prioritairement intéressée aux contenus, aux programmes mais plutôt aux objets eux-mêmes et à leurs interactions (déclarées) avec les utilisateurs.

2 Le travail de terrain s'est étendu sur deux années et nous avons travaillé avec une septantaine d'informateurs, tous habitants d'un village du Condroz belge. Cette localisation, parmi d'autres facteurs, donne à la recherche une "tonalité semirurale" qui apparaît comme une des caractéristiques spécifiques de la population avec laquelle nous avons travaillé. 
tique du contexte englobant et opère un découpage artificiel, en ce qu'il est effectué par une instance extérieure (le chercheur), et pourtant productif, en ce qu'il permet d'apporter des éléments de compréhension qui, s'ils sont déconnectés de la totalité, n'en demeurent pas moins articulés sur les représentations des informateurs.

Après avoir effectué un détour contextuel par la radio et la presse écrite, nous proposons deux axes interprétatifs de la construction domestico-médiatico-temporelle: la texture et l'occupation.

Insistons aussi, au regard de la spécificité de la population avec laquelle nous avons travaillé, sur le fait que le moment de la cessation d'activité professionnelle marque une rupture, un passage qui entraîne une reformulation de la situation, au sens d'Erving Goffman. C'est ce qu'exprime, au niveau du temps et de l'espace, Anne Wallemacq dans l'extrait qui suit et qui rappelle la position de Gilles Pronovost qui définit le travail comme "le temps-pivot"1 autour duquel les autres temps sociaux se déploient:

Tout se passe comme si l'opposition travail/loisir fonctionnait comme une balise temporelle, c'est-à-dire comme un code qui nous permet de nous repérer et de nous situer. C'est tellement vrai que lorsque cette balise disparaît, lors de la mise à la retraite par exemple, c'est non seulement tout le temps antérieurement consacré au travail qui devient problématique, mais aussi, ce qui en général est moins attendu par les intéressés, le temps antérieurement consacré au loisir. On observe qu'il perd sa consistance et que des activités anciennement fort appréciées perdent leur saveur, parce qu'elles ont perdu précisément leur statut de distraction par rapport au travail, par rapport à ce à quoi elles prenaient sens. Chacun des termes prend sens par rapport à l'autre. La mise à la retraite, du moins pour les travailleurs salariés, ôte sa pertinence à la structure qui a marqué des années durant la définition des temporalités et des espaces $^{2}$.

\section{a. Un détour contextuel par la radio et la presse écrite}

Je vais vous dire quelque chose: l'aîné de mes fils qui a 41 ans, il était tout petit et on écoutait assez bien la radio. Nous écoutions la radio, parce qu'une radio cela fait long-

1 G. Pronovost, "Le temps dans une perspective sociologique et historique", Revue intermationale des sciences sociales, ${ }^{\circ} 107,1986$, p. 9-10.

2 A. WALLEMACQ, "Le temps libre, une «uchronie»?", Belges, heureux et satisfaits (B. Bawin-Legros, K. Dobbelaere, J. Kerkhofs, L. VoyÉ, dir.) Bruxelles, De Boeck, 1992, p. 128-129. 
temps que nous en avons une. Eh bien, le gamin, il écoutait le marché à terme. Vous savez bien ce que c'est? Et quand on avait dit «financière de caoutchouc», eh bien, il pouvait partir [à l'école]. Il attendait ça tous les jours.

La radio, c'est, on le voit dans cet extrait d'entretien, une façon de "se donner l'heure", d'établir des repères qui permettent de s'accorder aux exigences sociales de la vie. Ainsi ces repères sont à la fois personnels, ("c'est après le journal parlé de neuf heures que je pars faire mes courses"), collectifs et sociaux puisqu'ils rattachent au monde en scandant, pour tous, le temps journalier qui défile tout en donnant à entendre un écho ou un murmure de la vie.

Pour beaucoup de personnes, la radio ponctue le début de la journée (la télévision étant plutôt la ponctuation de fin de journée):

Moi, je me lève et j'allume directement la radio qui reste souvent jusqu'après le journal parlé.

Quand on se lève, on va dehors nous, alors ... mon mari quand il rentre, il met ... la radio, ne fût-ce que pour déjeuner le matin.

En me levant, j'ouvre la porte, j'ouvre la radio, j'ouvre mes tentures ...

Je me lève et j'ouvre la radio ... C'est jusque quand on ouvre la télévision.

Ainsi la radio marque, en quelque sorte, l'entrée dans la journée, par une prise de température du monde, de la société, de la région. La radio est ponctuation d'ouverture, elle ouvre les guillemets de la journée. Plus tard, dans la matinée, elle offrira des balises pour les activités ("je dois avoir fait la chambre pour le journal de neuf heures") ou pour les rencontres ("le facteur arrive souvent après l'émission de musique classique à Bruxelles").

Par ailleurs, nous avons pu dégager, pour les couples, une priorité et une prédominance de la lecture masculine. En effet, le plus souvent, c'est l'homme qui lit le journal au moment où il arrive, c'està-dire en premier lieu, et c'est aussi lui qui le lit le plus longtemps. L'épouse, de son côté, le parcourt ou y jette un œil rapide le matin et le reprend parfois dans l'après-midi (sieste, après quatre heures). Nous n'avons relevé dans les descriptions qui nous ont été faites 
qu'une seule exception. Dans ce couple, c'est Madame qui lit le journal quand il arrive mais pour une raison bien particulière: elle est très malade et c'est Monsieur qui a pris en charge toutes les tâches ménagères. L'inversion se marque de façon encore plus claire quand Monsieur nous dit lire le journal pendant "l'après-dîner, un temps de repos où [il] ne dort pas". Nous pouvons avancer qu'il y a chez nos informateurs une répartition sexuelle de la lecture du journal. En ce qui concerne les revues, les choses sont quelque peu différentes dans la mesure où le temps de lecture ou de consultation (magazines de télévision) est plus long et où les revues ne sont, dans bon nombre de cas, lues que par un seul des membres du couple.

Autre tendance forte à se dégager de nos observations: la répartition spatio-temporelle de la lecture. Le matin, le journal se lit dans la cuisine et, le plus fréquemment, sur la table, après que celle-ci ait été débarrassée des "restes du déjeuner". Parlant du tapis qui est sur la table, une informatrice nous dit: "Regardez, le tapis à cette place-là, il est tout noirci. Regardez quelle différence! Et je ne sais pas le ravoir. Ça c'est l'encre du journal".

Ceci est surtout vrai pour les personnes qui reçoivent leur journal lors du passage du facteur. Ceux qui l'ont plus tôt à disposition le lisent, ou du moins le parcourent, déjà parfois, en déjeunant. Notons aussi que certains informateurs lisent le journal, le matin, dans la salle de séjour, là "où ils se mettent toujours pour lire ou regarder la TV".

En fin d'après-midi ou en soirée, c'est dans la salle de séjour qu'il est repris et, le plus souvent, à cette même place "où on se met" pour regarder la télévision, faire des mots croisés ou tricoter.

Entre ces deux moments de lecture, que nous retrouvons de façon très marquante chez la majorité des personnes rencontrées, le journal est déposé, en attente, à proximité d'un des lieux de lecture, sans que l'on puisse établir de similitudes précises quant à ces lieux. Ils peuvent se retrouver avec les autres revues, magazines et journaux sur une petite table, sur le coin d'un meuble, près d'un fauteuil, sur une chaise ou encore sur le bord de la cheminée. Ce qui importe, c'est que le journal reste disponible, à portée de main et consultable tant qu'il est encore d'actualité, c'est-à-dire avant qu'il ne soit remplacé par le suivant qui contient les dernières nouvelles et les programmes télévisés du jour. Ainsi soumis à un cycle rapide d'obsolescence, le journal est marqué -dans les propos de nos informateurs- à la fois, par la pérennité (le journal arrive chaque jour, se lit et se dépose aux mêmes endroits, renvoie aux mêmes habitudes) et le renouvellement 
(chaque jour un journál différent avec des nouvelles et des informations différentes).

Pour les revues et magazines, du moins ceux qui sont lus, nous avons constaté un même marquage fondé sur la pérennitérenouvellement qui se construit, cette fois, sur le rapport entre le terme de validité (la période pendant laquelle l'objet médiatique doit rester disponible) qui est endodéterminé et le cycle d'obsolescence (la fréquence de parution: semaine, quinzaine, mois) qui est exodéterminé ${ }^{1}$. Distinguons, dès lors, les revues "qu'on lit en plusieurs fois" ou "qu'on reprend plusieurs fois" (à titre d'exemple: les magazines de programmes télévisés, les news, les magazines féminins) où terme de validité et cycle d'obsolescence correspondent (comme pour les journaux quotidiens), et les "revues qu' on lit quand on les reçoit et qu'on ne garde pas" pour lesquelles le terme de validité est toujours inférieur et plus ponctuel que le cycle d'obsolescence (à titre d'exemple: "Touring-secours", les journaux mutuellistes, les revues et bulletins d'associations ou groupements). Nous proposons d'appeler les premières conchrones et les secondes dischrones. Bien que les exemples donnés traduisent assez bien les différenciations globalement opérées par les informateurs, il n'existe pas de partition modèle ou générale, chacun effectuant ses propres choix et fixant de la sorte, pour chaque revue, son propre rapport de validité en relation avec son cycle d'obsolescence. Bien que l'angle de vue soit différent -nous ne parlons ni de mode ni de valeur-, ce rapport entre terme de validité et cycle d'obsolescence rappelle ce que Jean Baudrillard mettait en évidence, quant au statut des objets dans le temps, sous le terme de "cycle d'usure et de renouvellement"2.

Notons encore, à propos des dons de journaux -assez fréquents dans le voisinage et dans la famille- et en restant dans cette même logique conceptuelle, que le prêt -soit un don temporaire- est de l'ordre de la conchronie (les deux usagers tentant de fonctionner sur la correspondance terme de validité-cycle d'obsolescence) tandis que le legs -soit un don définitif- est de l'ordre de la dischronie puisqu'au minimum un des usagers sera hors concordance.

1 Nous entendons par endodéterminé ce qui est fixé par l'usager et par exodéterminé ce qui est fixé par une instance extérieure à l'usager, le pôle de production en ce qui concerne les magazines et revues dont nous parlons.

2 J. BAUDRILlard, "La morale des objets. Fonction-signe et logique de classe", Communications, $\mathrm{n}^{\circ} 13,1969$, p. 39 et suivantes. 
Quand il n'y a plus aucune chance d'être pris pour être lu ou consulté, ou en d'autres mots quand il est hors terme de validité, c'està-dire quand il a perdu toute virtualité médiatique, le journal peut acquérir une virtualité domestique. On pourrait, à ce propos, parler de postqualification de l'objet ${ }^{1}$ qui, après lecture, sert à autre chose: récolter les épluchures de pommes de terre, protéger le pare-brise de la voiture, être utilisé comme litière pour le chat...

Quand le journal ou la revue a perdu aussi toute virtualité domestique, il est stocké avec ses semblables dans un endroit de rangement -cave, remise, garage- en attendant la collecte de vieux papiers organisée par les "Anciens prisonniers de guerre", car "voyez-vous, ici, on ne jette rien" et "ici, on ne brûle jamais rien".

On le voit la vie domestique des journaux et magazines s'organise selon un déroulement temporel, une chronologie répétitive qui fixe et localise tant les objets domestico-médiatiques eux-mêmes que les usages.

\section{b. Donner une texture au temps}

Regarder la télévision c'est d'une certaine manière rentabiliser un capital sans l'entamer car l'objet ne donne aucun signe d'usure, c'est gaspiller sans vraiment dépenser si ce n'est -ce point est d'importance- son temps ${ }^{2}$.

Le temps est précieux, il a de la valeur³. C'est à la fois ce que disent, dans la citation ci-dessus, Pierre Chambat et Alain Ehrenberg et ce que proclament nos informateurs qui, dans leur vie quotidienne, font des médias des instruments de gestion du temps et des donneurs de temps sociaux.

1 Ce terme est inspiré de F. Ponge (Le savon, Paris, Gallimard, 1967, p. 56) cité par M. Verret, (L'espace ouvrier, Paris, Armand Colin, 1979, p. 140) qui utilise le terme de "requalification" dans le sens de restauration de la valeur d'usage. Nous parlons plutôt de postqualification car il ne s'agit pas de restauration d'une valeur première mais d'émergence d'une valeur d'usage seconde.

2 P. Chambat, A. Ehrenberg, Télévision: essai d'identification d'un objet, Paris, Institut de recherche et d'information socio-politique, 1986, p. 45.

3 Dans le champ des études sur les médias, de nombreuses recherches ont porté sur une autre forme de valeur du temps, la valeur commerciale et marchande. C'est ce qu'évoque, P. BEAud (La société de connivence, Paris, Aubier, 1984) quand, faisant référence à des analyses antérieures, il écrit: "La radio et la télévision modifient pour l'usager l'idée de temps; elles monopolisent celui-ci, l'uniformisent en l'émiettant, et ceci dans un but: vendre du temps, vendre le temps des téléspectateurs" (p. 195). 
Partant des notions exclusives de monochronie et de polychronie qu'a proposées Edward T. Hall' et qui peuvent, selon lui, caractériser aussi bien des peuples (des cultures) que des individus, nous ne pouvons proposer une interprétation satisfaisante du rapport au temps de nos informateurs. En effet, ceux-ci ne sont ni strictement monochrones (compartimentage du temps, activité unique et multilocalisation des activités successives) ni strictement polychrones ("temps mélangé", multi-activité et localisation concentrée). Nous devons donc décloisonner et rompre l'exclusivité des notions défendues par l'auteur.

Ainsi, s'il apparaît bien que nos informateurs sont les "dignes fils" de la société industrielle en ce qu'ils valorisent globalement une logique monochrone (une seule activité en un seul temps en un seul lieu), nous devons cependant constater que la multi-activité n'est pas exclue en certaines circonstances et que la multilocalisation n'est pas une règle incontournable dans la mesure où une même pièce -en l'occurrence, la salle de séjour- peut trouver des affectations (souvent successives) différentes. Ainsi, il nous paraît plus pertinent de parler de monochronie ouverte, c'est-à-dire d'un rapport au temps délimité, réglé mais non strictement clôturé ou, en d'autres mots, d'une monochronie admettant (et même produisant) des zones polychrones.

A côté de cette ouverture, nous trouvons une crainte qui est souvent exprimée par les personnes du troisième âge: la peur du temps vide ou, de ce que nous appelons, la cénochronie. Quand il n'y a plus rien à faire, le temps perd toute valeur, il s'écoule, plat comme l'ennui, comme la mort. Notons que cette peur du vide est particulièrement forte au moment du passage à la retraite.

Ainsi, le temps de nos informateurs se trouve organisé "en segments qui reçoivent une affectation précise"2 ("Je me lève, je déjeune en écoutant la radio, je jette un œil sur le journal, je fais mes courses, je rentre préparer le dîner...") tout en évitant que des trous,

1 E. T. HALL, Le langage silencieux, Paris, Éd. du Seuil, 1984.

2 J. CAUNE, "Identité et culture: concepts fondateurs ou phénomène de mode?", Les cahiers du LERASS, n²3, 1991, p. 110. Cette segmentation n'entraîne pas, de notre point de vue, de rupture ni de perte de relation entre les parties qui se trouvent intégrées dans des logiques représentationnelles et les pratiques qui dépassent les compartimentages -en leur donnant du sens- et constituent l'ethos du groupe (tout en étant produites par celui-ci). 
des vides ne s'y installent. La meilleure façon de combattre ce danger est, alors, de donner du rythme ${ }^{1}$ au quotidien.

Cette monochronie ouverte, pleine et rythmée, nous proposons de l'appeler la texture du temps. Nous référant à Alfred Schutz, nous entendons par texture, à la fois l'état d'une chose tissée (travaillée) et la disposition des parties d'un ensemble'2.

La texture temporelle est un processus à la fois d'hétérogénéisation (des activités sont différenciées dans le temps et, sous diverses modalités, dans l'espace), de mise en liaison (ces activités se tiennent, se combinent les unes aux autres, prennent sens les unes par rapport aux autres) et de fixation (des usages-pivots émergent). Elle implique donc, dans le même mouvement, à la fois de la discrétion -au sens d'une certaine discontinuité-, de la linéarité -au sens de la continuité de ce qui défile- et de la périodicité -au sens de ce qui revient cycliquement-.

\section{c. Une temporalité de l'occupation}

Puisqu'on peut considérer que la notion de "temporalité" renvoie "aux représentations de la durée de processus spécifiques" et aux "modalités de la relation au temps"3, nous proposons de l'entendre comme la manifestation de ce qui existe dans le temps ou, plus précisément, comme le mode de consommation-production (l'institution symbolique) du temps. Parler de temporalité, c'est envisager le temps en tant qu'élément culturel ${ }^{4}$ et défendre l'idée que toute culture temporelle est marquée contextuellement, entre autres, par l'histoire et

1 "Rythme" au double sens proposé par D. MERCuRE ("L'étude des temporalités spatiales", Cahiers internationaux de sociologie, vol. LXVII, 1979, p. 268 et suivantes) de ce qui est de l'ordre de la périodicité, de la continuité, de la "succession des régularités" et de ce qui est de l'ordre du changement, de la vivacité.

2 A. SCHUTZ, Le chercheur et le quotidien, Paris, Méridiens Klincksieck, 1987. L'auteur écrit (p. 14) que la culture est une texture signifiante.

3 C. LALIVE d'EPINAY, "Le temps et l'homme contemporain", Les temps sociaux (D. Mercure, A. WallemaCQ, dir.), Bruxelles, De Boeck, 1988, p. 18.

4 "Du point de vue socio-anthropologique, on considère généralement que le temps fait partie de la culture et relève de la sphère culturelle au sens où il représente une construction sociale typique et fondamentale de la réalité, œuvre des systèmes et des acteurs qui en sont partie intégrante" (G. GASPARINI, "Quelques observations sur les modèles culturels du temps dans les sociétés industrialisées contemporaines", Information sur les sciences sociales, tome $29, \mathrm{n}^{\circ} 4,1990$, p. 725). 
la surmodernité de nos sociétés, pour reprendre une expression de Marc Augé .

De ce point de vue, il nous semble que les médias domestiques peuvent être comparés à l'horloge, telle que Giovanni Gasparini en définit les fonctions:

Dans les sociétés industrielles, l'horloge a deux fonctions fondamentales, liées entre elles: la synchronisation interpersonnelle et sociale, d'un côté, et, de l'autre, l'intériorisation par les individus des cadres temporels ${ }^{2}$.

Des chercheurs, comme Gilles Pronovost, ont tenté de mettre au jour diverses temporalités sous-jacentes aux interactions consommateurs / médias ${ }^{3}$ : temporalité de l'instantané / temporalité de la durée, temporalité centrée sur la sphère privée / temporalité centrée sur la sphère publique, temporalité d'une consommation passive / temporalité d'une consommation active... Reprenant cette dernière opposition, nous proposons de la rapprocher de ce que disait Anne Wallemacq de l'ennui et de l'agitation 4 et de l'implanter dans notre réflexion. Ainsi à une temporalité de l'ennui (ne rien avoir à faire) et à une temporalité de l'agitation (avoir trop à faire), nous souhaitons ajouter un troisième mode d'institution symbolique du temps, que nous appelons l'occupation.

Pour nos informateurs, "il y a toujours quelque chose à faire", "il y a toujours moyen de s'occuper", "il faut toujours trouver quelque chose à faire". Nous avons pu mettre en évidence que leur pratique

1 Par la notion de "surmodernitê", Marc Augé désigne les "effets d'accélération, de trop plein et d'excès qui, loin d'abolir ou de dépasser la modernité telle qu'on la concevait au $\mathrm{XIX}^{\mathrm{e}}$ siècle, la surdéterminent, la rendent du même coup moins lisible et plus problématique" (Colloque Sports, médias et société, Louvain-la-Neuve, le 8 mars 1995).

2 Ibid., p. 736. Nous retrouvons ce même rapprochement -jugé de façon, parfois, radicalement différente- chez d'autres auteurs, comme S. BLUM (La télévision ordinaire du pouvoir, Paris, PUF, 1982) qui écrit: "A l'instar de l'horloge, la grille télévisuelle, projection spatiale du temps, impose ainsi à chacun une mainmise déterministe et rassurante sur son devenir. Le télévisuel est, semble-t-il, la première substance d'expression qui intègre organiquement le temps, temps chronologique, qu'elle prévoit, qu'elle divise et intensifie" (p. 138). J. PERriault (La logique de l'usage, Paris, Flammarion, 1989) écrit, de son côté, que la télévision devient "l'horloge contemporaine qui règle les temps de la vie quotidienne" (p. 163).

3 G. Pronovost, op. cit.

4 A. WallemacQ, L'ennui et l'agitation. Figures du temps, Bruxelles, De Boeck, 1991. 
domestico-médiatique, et singulièrement leur pratique télévisuelle, se donnait à comprendre sous la double logique de l'intérêt (comprenant le plaisir et la détente) et de la conscience et qu'il n'était pas question "de se laisser manger par la télévision". La télévision prend donc place dans les activités et dans le "temps ordinaire" de la journée à côté d'une série d'autres activités. Les propos qui suivent, même s'ils ont quelque chose d'excessif', reflètent assez bien l'impression générale qui se dégage de l'ensemble des réflexions de nos informateurs, pour qui l'occupation s'impose culturellement comme un mode de consommation-production du temps domestique 2 :

Je ne fais rien pour passer mon temps. Parce que le temps passe trop vite. Je fais ce qui doit être fait. Moi, faire quelque chose pour passer mon temps, c'est... c'est en prison qu'on fait quelque chose pour passer son temps. Quand j'étais en captivité, j'ai fait quelque chose pour passer mon temps. Il y a des gens qui disent parfois: comment ne viens-tu pas jouer aux cartes? Ah non, je ne fais rien pour passer mon temps. Nom di Dieu, j'sais bien, il passe vite le temps. Je fais ce qui doit être fait. Je taille mes rosiers; je taille mes arbres, si ça doit être fait. Mais je ne fais rien pour passer mon temps. Parce qu'il me semble que c'est une vilaine réflexion, ça, faire quelque chose pour passer son temps. Le temps, ça ne m'ennuie pas.

Pour nos informateurs, il n'est pas question de se laisser aller à des activités cénochrones, qui ne sont qu'une façon de déguiser du temps vide, du temps mort. Ainsi, même ceux qui disent regarder la télévision quand ils ne savent trop quoi faire (quand il fait froid, quand il pleut, quand ils sont moins bien physiquement), par la conscience qu'ils en ont, instituent cette pratique comme une véritable occupation, de dépit peut-être, mais occupation tout de même. Nous retrouvons peut-être là, dans une dimension représentationnelle (c'est-

1 Excessif, dans la mesure où, pour beaucoup d'autres informateurs, participer à des réunions ou jouer aux cartes sont des activités à part entière, de véritables occupations (pas des passe-temps dérisoires et cénochrones) comme l'est le jardinage pour cet informateur.

2 Il nous semble, par ailleurs, que cette temporalité de l'occupation déborde du cadre strictement domestique pour s'imposer plus globalement dans l'ensemble de la vie quotidienne. Différents indices qui réfléchissent (reflètent, font advenir à la conscience et construisent socialement) la vision qu'ont nos informateurs des activités et de la temporalité extra-domestique, vont dans le même sens et portent sur les voyages, les visites ou les services à la famille, les courses et les activités culturelles, les engagements associatifs... 
à-dire à la fois personnelle et en continuelle construction sociale), ce que Mircea Eliade appelle "les résidus d'un comportement mythologique": "Comme il fallait s'y attendre, c'est toujours la même lutte contre le Temps, le même espoir de se délivrer du poids du "Temps mort", du temps qui écrase et qui tue"'.

Expression de la maîtrise et de la conscience des choses, cette temporalité de l'occupation n'est ni une temporalité en suspens ni une temporalité du strict comblement. Pour aucun de nos informateurs, les activités domestico-médiatiques (essentiellement celles qui sont liées à la télévision ou au journal) n'apparaissent comme des pratiques "de troisième zone" auxquelles ils ne tiennent pas tellement et qui ne sont là que parce qu'ils n'ont aucune autre possibilité. Au contraire, les pratiques domestico-médiatiques sont revendiquées comme partie prenante de la structuration et de l'organisation temporelle.

La vision d'une télévision omniprésente, totalitaire, où le temps de l'émetteur serait seul structurant, est loin de la représentation qu'en ont -qu'en donnent- nos informateurs. D'autre part, la pratique télévisuelle prend sens non plus comme pratique de loisir par rapport au travail (considéré comme temps-pivot) mais comme pratique particulière d'intérêt (documentaire ou distractif) par rapport aux autres occupations.

Ces représentations partagées, intersubjectives appellent certaines pratiques (du moins, dans l'expression sociale qui en est donnée) qui sont reconnaissables et donc évaluables pour soi comme pour les autres.

\section{d. Quand le temps s'inscrit dans l'espace}

Pour conclure cette première partie de l'article consacrée aux temporalités vécues et en insistant sur l'importance des effets discriminants de l'âge sur les pratiques, nous pourrions dire que les pratiques domestico-médiatiques participent activement à la constitution d'une temporalité de l'occupation en ce qu'elles donnent un cadre temporel (fixent des repères et des balises), donnent corps, consistance au temps et donnent à partager avec d'autres, non pas simplement le fait synchronique d'être branché en même temps sur le même programme, mais, plus fondamentalement, un même rapport constitutif au temps.

1 M. EliAdE, Aspects du mythe, Paris, Gallimard, 1975 (1 ère éd. 1963), p. 232. 
Bien que n'ayant pas véritablement traité, dans ce qui précède, des temporalités propres aux mass médias ni des "horizons temporels" (intégrant la reconstruction du passé et l'anticipation de l'avenir) qu'ils embrassent ni de ce que nous pourrions appeler des temporalités contextuelles, il nous semble, néanmoins, que nos informateurs ne se représentent (et, ce faisant, ne construisent) pas le temps, leur temps de façon disloquée, décousue. "Coutumier, intimement vécu, le temps n'apparaît pas d'emblée comme un problème"1, dans la mesure où il demeure et s'inscrit dans le champ de maîtrise effectif-déclaré de nos informateurs âgés et s'institue, de la sorte, symboliquement.

Par ailleurs, il nous paraît évident que, dans la sphère domesticomédiatique, l'espace et le temps entretiennent des rapports étroits et différenciés qui font système. Pour les individus, ils prennent forme l'un par l'autre, l'un dans l'autre. Le temps de la journée qui défile $s$ 'inscrit dans des configurations spatiales et des parcours répétés au fil des jours. La temporalité de l'occupation trouve à "s'incarner" dans des pratiques, entre autres médiatiques, localisées dans l'espace de résidence ou à l'extérieur, de telle sorte que le plus insaisissable trouve à s'instituer dans du manifeste et que le temps se trouve appréhendé, entre autres, par des modes d'appropriation de l'espace domestique.

"Le chez-soi prend consistance dans la régularisation des rythmes des activités domestiques, dans la répétition inlassable des gestes du quotidien ou dans les effets de synchronisation et de mise en phase de pratiques diachroniques ou conflictuelles"2 et, pourtant, intégrées. Les pratiques domestico-médiatiques scandent, organisent, architecturent, remplissent, constituent le temps quotidien. Le passage du facteur, la visite chez le voisin à qui on prête le journal, les informations de 13 heures à la radio et, surtout, les émissions -dont le JTdu début de soirée à la télévision... sont autant de repères qui vont baliser la journée en affectant des mobilisations configurationnelles spatiales particulières à des périodes ou à des moments spécifiques marqués par des activités singulières qui vont donner au temps une certaine consistance occupationnelle.

1 R.-P. Droit, "Le lieu du temps", Le Monde, 13-11-92, p. 40 (à propos de J.-T. DESANTI, Réflexions sur le temps: variations philosophiques I, Paris, Grasset, 1992).

2 P. Amphoux, L. Mondala, "Le chez-soi dans tous les sens", Architecture et comportement, vol. $5, \mathrm{n}^{\circ} 2,1989$, p. 143. 


\section{Temporalités conçues}

Après avoir réexploité, de façon indépendante, certains éléments de deux recherches empiriques, nous souhaitons maintenant cerner quelques modes d'appréhension du temps médiatique. Loin d'épuiser une trop riche matière, nous nous limiterons aux références et aux observations qui apparaissent les plus adéquates par rapport à notre question de départ. En d'autres mots, nous allons tenter de percevoir comment la programmation et les logiques de production télévisuelles sont traversées, transpercées par (les représentations de) la réception.

\subsection{Temporalité et texte télévisuel}

En se référant au procès de réception et à chacun de ces éléments -le téléspectateur, le texte, la relation que le premier entretient avec le second-, il nous apparaît que la dimension "temporalité du média" est sous-jacente à l'ensemble des analyses et débats récents relatifs à la nature du texte télévisuel. Il est intéressant de noter qu'au-delà des différences d'approche existant en particulier entre les narratologues, les sémioticiens et les chercheurs en communication, tous s'accordent actuellement sur le caractère particulier de la télévision sinon en tant que texte du moins en tant que forme.

L'étude de la télévision, dans son vocabulaire et ses méthodes d'analyse, s'est bien sûr nourrie de la théorie du cinéma, mais l'extrapolation de la notion de texte de l'œuvre cinématographique au programme télévisuel ne s'en voit pas moins irrémédiablement récusée par maints auteurs. Par contre, de récents travaux, considérant comme erronée une opposition entre petit et grand écran, "débordent" de l'analyse des programmes et privilégient une analyse de la forme télévisuelle à un niveau plus global, en particulier celui de la grille.

La télévision n'est ni une annexe ni une perversion du cinéma, elle est un autre système d'écriture; au cinéma elle emprunte une grande partie de ses matériaux et de ses techniques mais sa logique est tellement différente qu'il serait absurde de vouloir l'évaluer à la dimension du septième art ${ }^{1}$.

1 P. Sorlin, Esthétiques de l'audiovisuel, Paris, Nathan, 1992, p. 181. 
Pour ces auteurs, la caractéristique majeure de la télévision, quelles que soient les formes antérieures auxquelles elle puisse se référer, est son inscription dans une logique propre, une logique de continuum sans commencement ni fin. Les messages se succèdent alors dans un flux permanent et la télévision "s'affirme par sa seule permanence, elle est une narration continue" .

Que les travaux des différents courants de recherche accordent la prééminence à cette notion de flux télévisuel -flux qui se voit ordonné dans une grille- ou se consacrent aux seuls programmes, cette dimension de la forme télévisuelle, tout comme sa conséquence première, l'empreinte dont elle marque chacune des unités qui la composent, sont prises en compte par tous les auteurs. Dans ce contexte, certains d'entre eux nous mettent, toutefois, en garde contre la tendance forte à générer, à partir de cette notion de flux, uniquement des discours globalisants sur l'ensemble de la télévision, occultant de ce fait la diversité des modalités de réception induites par le système des genres, même si celui-ci se trouve actuellement mis à mal.

\subsection{Le flux télévisuel: plus qu'une simple succession de séquences}

La succession ininterrompue des émissions, nous l'avons évoqué, introduit une certaine "porosité" de chacun des éléments constituant l'enchaînement des programmes, d'une journée par exemple. Les séquences interagissent avec le flux dans lequel elles s'insèrent et chacune d'entre elles voit ses frontières se défaire sinon dans un texte unique du moins dans une continuum toujours discernable. Pierre Sorlin définit dans ce sens les traits caractéristiques des diffusions télévisuelles, sans adopter la thèse extrême d'une totale dissolution du programme dans le flux qui le porte.

Il s'agit en premier lieu d'unités standardisées, à peine distinctes, substituables les unes aux autres; ces pièces sont conçues de façon à se regrouper, de plusieurs manières différentes, en ensembles plus vastes; de là provient une tension permanente entre une totalité qui n'intègre pas totalement ses parties et des éléments singuliers mais trop peu autonomes pour se manifester seuls, en dehors de l'ensemble. Cette rela-

1 Ibid., p. 193. 
tion instable caractérise relativement bien les programmes télévisuels... Elle permet de mieux voir pourquoi le petit écran met à mal le film entendu comme œuvre, sans nécessairement modifier sa texture audiovisuelle; par rapport aux choix que le spectateur opère dans la "grille", le film est ce que l'on passera à telle ou telle heure, il remplit une case de programme, et, à cet égard, ne se distingue pas de n'importe quelle autre réalisation (...). Le film n'est pas condamné (...) mais, à son individualité d'œuvre close, la télévision juxtapose, désormais, un au-delà de l'œuvre'.

\subsection{La grille: structure textuelle et/ou temporelle, un problème de rythme}

Nous allons nous arrêter à deux approches de la grille des programmes, en tant qu'ordonnatrice du flux télévisuel. La première, celle de Dominique Chateau ${ }^{2}$, s'interroge sur la pertinence à la reconnaître ou non comme une structure textuelle et la seconde, celle de Nora Rizza ${ }^{3}$, travaillant sur la programmation des chaînes italiennes et considérant que la temporalité est une dimension constitutive de la communication télévisée, met en avant l'analyse de la grille comme "modèle temporel".

Pour Dominique Chateau, la notion de texte télévisuel ne doit pas se voir définitivement récusée du seul fait que la forme générale du conditionnement du programme télévisuel n'est pas assimilable à celle de l'œuvre cinématographique. S'il semble manquer à la télévision "la principale propriété que l'on associe généralement à l'idée de texte: la forme organique qui assure la liaison prétablie et définitive des éléments d'un livre ou d'un film en sorte qu'on peut relire l'un et revoir l'autre, tels quels, à maintes reprises", il considère toutefois que la construction du flux de programmes autorise une approche textuelle.

A l'émission comme à la réception du message télévisuel, on parle de grille de programmes pour désigner à la fois la forme

Ibid., p. 207.

2 D. Chateau, "Horlogisme ou la télévision comme forme", Médias et information, $\mathrm{n}^{\circ} 1,1993$.

3 N. RizzA, "Stratégies de programmation dans la logique de concurrence: le cas italien", Colloque Sociologie des télévisions en Europe, Paris, Centre Georges Pompidou, 24-25 janvier 1991. 
et le contenu de leur enchainement; dans un cas comme dans l'autre, ce terme désigne une énumération ordonnée de messages. Parler en l'occurrence de structure textuelle, ce serait supposer que cette grille, par-delà la juxtaposition linéaire qu'elle déploie, comporte une structure à la fois logique et chronologique d'où s'en suivraient des relations d'ordre proche et lointain, des rétroactions et des anticipations des programmes les uns sur les autres. Certains de ces effets sont bien connus...

Une analyse comparative des formes des grilles américaine et française conduit D. Chateau à l'appréciation suivante: la forme américaine mettant en ouvre une plus grande régularité du rythme de la grille apparaît plus conforme que son équivalent français -où, au-delà des rendez-vous et des habitudes, règne une marge d'incertitude liée à une proportion plus large de prestations en direct- à sa conception de structure textuelle.

Pareille restriction à la compacité a priori ${ }^{2}$, à des programmes prévus et mesurés d'avance va de pair avec une automatisation généralisée des programmes qui a pour conséquence essentielle de rendre possible la maîtrise à la seconde près de leurs enchaînements, le respect scrupuleux de l'horaire annoncé et l'encadrement du téléspectateur par un continuum uniformément rythmé(...). L'horlogisme télévisuel est assorti à une forme de trame où la gestion de la temporalité semble avoir davantage d'importance que le contenu des programmes; la régularité de la fragmentation des programmes et de la répétition des messages intercalaires impose une loi formelle qui rétroagit sur le contenu des uns et des autres ${ }^{3}$.

Les travaux de Nora Rizza n'abordent pas les effets du degré de "compacité" des programmes sur l'occupation de la temporalité télévi-

1 D. ChATEAU, op. cit., p. 32.

2 "J'appelle message compact a priori un programme préfabriqué dont le passage à l'antenne se limite à sa diffusion tel quel, message compact a posteriori, un programme dont la compacité s'en suit de sa diffusion et message mixte, un programme qui comporte des parties appartenant à chacune des catégories. Le film appartient à la première catégorie, un programme sportif à la seconde, le journal, où la prestation en direct du journaliste est entrecoupée de messages préfabriqués, à la troisième.... En d'autres termes, la compacité désigne une propriété globale de la diffusion et non du contenu des programmes" (Ibid.).

3 Ibid., p. 35. 
suelle, mais ils rejoignent ceux de Dominique Chateau quant au rôle déterminant de la grille comme mode de gestion de cette temporalité.

Dans la logique de concurrence qui régit l'actuel système télévisuel (chute des différents monopoles de service public, multiplication des chaînes commerciales, poids des recettes commerciales dans les revenus des sociétés de télévision...), Nora Rizza analyse, à partir de l'exemple italien, le rôle stratégique joué par la programmation: organiser l'offre, orienter la consommation, "exploiter le temps d'écoute". Le véritable enjeu de la perpétuelle compétition entre les chaînes du moment n'est autre que le temps des téléspectateurs qu'il faut tenter de mobiliser. Les dispositifs mis en place visent à favoriser des habitudes d'écoute, à créer des attentes, à imposer par la facture des émissions offertes une image de la chaîne; "à la fin, la grille des programmes ne sera pas seulement la somme des éléments dont elle est composée"1. Pour cet auteur, l'analyse de son contenu ne peut rendre compte, dans un tel cadre, de la logique de la construction du flux des programmes, aussi aborde-t-elle la grille comme une structure textuelle, la grille étant tout à la fois le "texte global" mis en œuvre par l'émetteur et "le contexte qui structure et organise d'une manière syntaxique les unités de programmation, les conjonctions et la ponctuation (...). Elle est le calendrier et l'horloge de ce temps télévisuel, dont elle organise et indique les durées, les rythmes et les répétitions"2.

"Rythmer, ce n'est pas seulement ordonner, c'est chercher à entraîner quasi biologiquement"3. Faire coïncider au mieux les rythmes du flux des programmes avec les rythmes domestiques, le temps télévisuel avec celui de la vie quotidienne se révèle l'exigence qui sous-tend toutes les stratégies des chaînes généralistes. "Accaparer le temps des téléspectateurs, pas seulement le temps des loisirs mais aussi le temps social de la quotidienneté"4 est une des caractéristiques de la relation que la néo-télévision établit avec son public.

\footnotetext{
1 N. RizZA, op. cit., p. 294.

2 Ibid., p. 299.

3 F. BRUNe, Les médias pensent comme moi, Paris, L'Harmattan, 1993, p. 30.

4 N. Rizza, op. cit., p. 296.
} 


\subsection{La programmation structurée par la quotidienneté}

Cette mutation de la relation émetteur-public inhérente au "passage de la paléo-télévision à la néo-télévision", selon la fameuse formule d'Umberto Eco, recouvre d'autres dimensions. Nous retiendrons ici celles qui participent à mettre en nuvre un "média de quotidienneté", à "décalquer" la grille sur les rythmes de la vie domestique. "La télévision, tout en considérant le quotidien comme un modèle, devient à son tour modèle du quotidien"l, constatent R. De Berti, A. Negri et P. Signorelli, dans leur analyse des caractéristiques de la néo-télévision.

L'une des caractéristiques majeures de ce passage est, dans une certaine mesure, la perte du caractère événementiel dominant de la télévision (il n'a, bien sûr, pas totalement disparu et trouve même des formes et des voies nouvelles d' "exploitation"). Cette perte, liée à la multiplication de l'offre, induit une banalisation du média et de la relation que le public entretient avec lui mais, également, l'évolution interne de la télévision. Elle se rapproche du récepteur à la fois par ces contenus et par un brouillage de la relation émetteur/téléspectateur, scène/salle, la possibilité de passer soi-même derrière l'écran, comme public ou acteur, devenant plausible par le biais de nombreuses émissions de plateau. Dans ce contexte, les frontières entre les genres de programmes ont tendance à s'estomper. "La télévision de l'ère du monopole tendait à organiser son rapport avec le public en distinguant clairement et nettement les types de programmes"2. C'est essentiellement à partir des modalités et des règles des genres que se structurait la relation entre la télévision et son public. Les genres suscitaient l'adhésion. Aujourd'hui, la majorité des programmes "ne sont plus pensés comme des événements uniques mais, plutôt, comme des rencontres fixes et ouvertes (...) avec des caractéristiques de tous les jours"3. Ces programmes visent à instaurer des liens fortement individualisés avec chacun des téléspectateurs, à créer un nouveau rituel basé non sur la "consommation festive" mais sur l'habitude et "construit sur la répétition". De cette façon, "la télévision englobe en son sein la ritualité quotidienne au niveau des modalités de la représentation. De plus, en assumant la ritualité quotidienne, elle lui resti-

1 R. De Berti, A. Negri, P. Signorelli, "Scènes de la vie quotidienne", Réseaux, $\mathrm{n}^{\circ} 44-45,1990$.

2 Ibid., p. 59.

3 lbid., p. 60. 
tue un forme d'ordre télévisuel et construit le pacte avec le téléspectateur'l.

L'éternelle quête d'un rapport interactif fort et continu avec le public conduit à promouvoir des formes nouvelles, à métisser les genres; la notion de genre perdant, de la sorte, de plus en plus son sens. Alain Le Diberder qualifie le phénomène d'hybridation. "Mon idée, dit-il, c'est qu'on va plutôt vers des émissions hybrides qui mélangent des choses qui peuvent être très éloignées, comme le documentaire, le jeu et la variété"'.

Ce métissage se retrouve également au niveau du contenu. La distinction conventionnelle entre réalité et fiction s'estompe également dans de nombreuses émissions. Certes, cette manifestation n'est ni nouvelle ni récente. Raymond Williams l'évoque à propos d'une forme de "fiction-documentaire" dont il situe l'émergence dans les années soixante-dix en Angleterre et aux États-Unis. Il la définit comme "le mélange intrinsèque des conventions du reportage et de la fiction dramatique"3. Mais cette interpénétration réalité/fiction et la "fictionalisation" du réel sont aujourd'hui plus fréquentes, en particulier dans les reality shows. Les "grands récits" laissent de plus en plus fréquemment la place aux histoires du quotidien qui cherchent à s'inscrire directement dans la sphère privée des téléspectateurs.

\subsection{Le flux non dévoré par le genre}

Que reste-t-il, dès lors, de la narration dans cette télévision à la recherche du quotidien qui semble éloigner le récit de l'écran? Beaucoup de programmes télévisés -au-delà des films diffusésadoptent la forme du récit. Malgré ce constat, Pierre Sorlin considère que pour une télévision inscrite dans une logique de permanence "partager le domaine audiovisuel entre les récits et les non-récits serait pourtant une erreur [car] ces deux catégories relèvent également de l'effet narratif au sens large, il s'y opère un transformation au fur et à mesure qu'elles se déroulent"4.

1 Ibid., p. 64.

2 Entretien avec Dominique MEHL, in La fenêtre et le miroir, la télévision et ses programmes, Paris, Payot, 1992, p. 56.

3 R. Williams, "Television, Technology and Cultural form", Réseaux, $\mathrm{n}^{\circ} 44-45$, 1990.

4 P. SORLIN, op. cit., p. 190. 
Certains auteurs déplorent qu'une utilisation excessive voire exclusive de la notion de flux aboutisse pour nombre d'études de réception -mais également d'études textuelles- à une "version homogénéisée de la télévision". Pour John Corner, "la notion de flux télévisuel commence par être une notion d'analyse textuelle, une observation de phénomènes formels mais elle glisse en douceur vers une théorie de la réception et de l'influence sans qu'à aucun moment une reconceptualisation ou une preuve empirique ne soit considérée nécessaire". La résistance à cette version dominante d'une "essence de la télévision" passe, pour lui, par un retour à la notion de genre, à la reconnaissance de la différence générique. Il ne s'agit pas, cependant, pour l'auteur d'examiner le genre comme une forme figée, mais d'analyser la façon dont il peut relier entre elles différentes dimensions attribuées traditionnellement à tel ou tel genre, de mettre en lumière des facteurs "intergénériques" qui interviennent lors de la réception par le public.

Roger Silverstone, pour sa part, s'insurge face au consensus existant pour traiter les genres spécifiques du média télévision comme des "formes discrètes". Il défend que des genres ne dépendant pas nécessairement du cadre de la narration classique (tels les journaux télévisés qui fournissent de la matière aux interactions quotidiennes, les soap-operas..) "participent au travail essentiel de médiation qui fait de la télévision le lieu d'une composante distincte et continuelle de la culture, (...) à peine séparés du mythe et de l'interdépendance étroite que toutes les formes de narration télévisée ont avec le quotidien"2.

\subsection{Image et son: une double temporalité}

Toute stratégie de programmation d'un média présuppose certaines modalités de consommation. En fonction des moments de la journée, les programmes tenteront soit de s'imposer aux habitudes et aux rythmes de la vie quotidienne soit de s'y insérer. Ainsi, la relativement récente télévision du matin, à forte dominante radiophonique, peut être associée, comme nous l'avons évoqué dans la première partie de cet article, avec des activités d'une plage horaire ne

\footnotetext{
$1 \mathrm{~J}$. CoRner, "Genres télévisuels et analyse de la réception", Hermès, nº11-12, 1992.

2 R. SiLverstone, "Télévision, mythe et culture", Réseaux, n44-45, 1990.
} 
demandant pas un attention visuelle continue. Cette forte distance entre vision et écoute n'est pas propre aux formes télévisuelles sollicitant davantage l'oreille que l'cil, elle n'a rien d'exceptionnel. Elle participe de la gestion constante de deux temporalités contrastées, celle prolongée du discours, celle quasi immédiate de l'image. Pour Pierre Sorlin, dans un modèle où "le télévisuel standard est fait pour qu'on entende sans nécessairement voir, (...) le rapport image/son n'est pas de l'ordre de la complémentarité, il ressortit plutôt à la substitution. Le verbal rassure par son caractère uniforme, il engendre une écoute vague qui, à partir d'un changement de ton, d'un silence, deviendra plus attentive. L'image, immuable dans sa construction, insignifiante, semble d'abord invisible; il suffit que son architecture interne se dérègle ou que le son vacille pour que, soudain, elle attire l'œil"1. Il voit dans le clip, forme emblématique de la réalisation télévisuelle, une totale autonomisation des deux régimes temporels, le son pouvant exclure l'image et réciproquement. "Le clip évolue entre deux extrêmes qui sont le redoublement du son par la vue ou la mise en conflit des deux registres sonore et visuel. (...). Le public jouit alors d'une faculté supplémentaire, il choisit sa forme d'adhésion"2.

Serge Daney rejoint cette analyse de Pierre Sorlin sur les séquences en "direct" -enserrées dans des programmes que Dominique Chateau qualifiait de compacts a priori- où "la durée perd son élasticité". Il lie sa perte du "désir de voir" à cette connaissance, également a priori, de la durée du spectacle. "Si nous faisons un effort, nous nous rendons compte qu'à la télé, nous savons par avance toutes les durées et que ce savoir-là décourage -avant même qu'il ait été mis en branle- le plaisir de voir. Le plaisir le plus extraordinaire est cette drôle d'intonation dans les phrases des présentateurs du JT qui annoncent que ce qu'ils disent sera immédiatement suivi d'un petit reportage en images. Le parlé fait la chaîne (ou la courte échelle) au montré sous la surveillance du spectateur qui, du coup, regarde moins ce qui s'inscrit dans la durée qu'il ne vérifie celle-ci"3.

Pour Alain Gauthier", "l'effet écran, l'effet de mobilisation, l'effet expérimental, l'effet de serre mentale, l'effet d'urgence [sont autant d']

1 P. SORLIN, op. cit., p. 173.

2 Ibid.

3 S. DANEY, Devant la recrudescence des vols de sacs à main, Paris, Aléas Editeur, 1991, p. 78-79.

4 A. GAUTHIER, L'impact de l'image, Paris, L'Harmattan, 1993. 
effets qui viennent prolonger la capture du regard par le système image". Partant de cela, l'auteur dégage trois catégories d'images. L'image "endémiquel se caractérise par son extrême redondance (...) et son extrême capacité à la duplication". Son rapport au temps se caractérise par la rediffusion, le retour du même. L'image "endémique met à l'abri du temps", elle efface son empreinte. Avec l'image fulgurante, la télévision joue sur un autre aspect du temps, "l'instantanéité". "C'est brancher l'événement sur le médium," c'est le scoop, l'image inattendue. L'image par son intensité brûle toutes les autres appréciations (sur la catastrophe, la misère...) car elle isole du contexte (...). Le temps de l'image se plie au principe de vitesse pour imposer le modèle de l'urgence. Le flux-image par son caractère phatique retombe sur l'audience, offre une vision surplombée des choses jusqu'à ce qu'elles deviennent inopérantes dans leurs effets (pour leur dimension fulgurante) et dans leur durée (pour leur aspect endémique)".

Alain Gauthier définit alors l'“état d'abandon" comme une des réponses du public face à la surabondance d'images, comme "une sorte de hors-temps qui permet de se dégager de la pulsation chronique de l'image, tout en y participant". L'abandon se pose comme le gardien du temps, un état de veille malgré tout attentif, "à la manière d'un chat qui, apparemment ensommeillé, semble toujours prêt à réagir à la proie qui se présenterait accidentellement dans son champ de vision".

\section{Le téléspectateur face à une nouvelle donne}

Cadre du message télévisuel à l'émission comme à la réception, la grille du "programme global" est, selon la formule vigoureuse de De Berti et son équipe, "une véritable arène de la négociation". Pour eux, nous l'avons évoqué, le nouveau pacte avec le téléspectateur est

1 Moins souvent diabolisée que l'image fulgurante, l'image endémique, "matériau indépendant de l'évenement", peut se voir identifiée et dénoncée; ici, par le critique de programmes de télévision du Canard enchainé, Yvan Audouard. A propos d'images représentant $M$. Jacques Médecin en liberté à Punta del Este, alors qu'il est incarcéré et à la veille de son extradition en France, il conclut par ces phrases sa chronique, "La Boîte à Images", intitulée ce mercredi-là, 26 octobre 1994, "Trompe-Couillons": "La télé est devenue, dans tous les sens du terme, une «machine à tuer le temps». Car elle vous rend incapable de distinguer le passé de l'avenir. Ce qui permet à n'importe quelle cause de produire n'importe quels effets". 
basé sur une ritualité quotidienne. "La ritualité est à la fois le signe du pacte et le lieu du pacte". Ils construisent un modèle qui articule la quotidienneté dans le flux télévisuel selon des rituels communicatifs, des modèles différents de gestion du flux qui peuvent se mélanger dans des programmes faisant appel à des stratégies différentes.

Mais que nous dit cette conceptualisation des stratégies de programmation et des pactes communicationnels d'une télévision qui prétend prendre en compte la vie du récepteur dans tous ses moments et dans toutes ces aspirations? Rien ne nous autorise à décider "si c'est un type de structuration identique qui préside à la réception" comme le déplore Pierre Sorlin à propos de la narratologie. Rien ne permet d'affirmer que les mécanismes effectivement repérables dans le texte aient une fonction déterminante pour sa lecture.

L'évolution qualitative et quantitative de l'offre télévisuelle européenne se caractérise par la coexistence d'une part de chaînes généralistes et d'autre part de chaînes thématiques et spécifiques (Arte, télévisions locales et régionales) inscrivant intentionnellement leurs programmes dans une structure temporelle différente de celle des chaînes généralistes classiques.

Dans ce contexte, où une partie de l'offre ne vise pas à faire coïncider rythmes de la grille et rythmes de la vie quotidienne, les modèles de la relation public-média -du modèle du "rendez-vous" au modèle du "flux" en passant par les figures intermédiaires mêlant les deux modèles contrastés-, les appropriations, les pratiques et les représentations des récepteurs doivent être (ré)interrogées. 